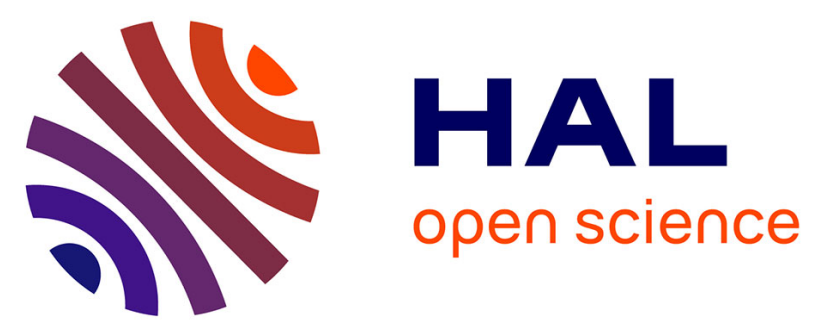

\title{
Quelle information accompagne la distribution des Compléments Nutritionnels Oraux (CNO) à l'hôpital : un état des lieux
}

Marie-Claude Brindisi, Ousrat Chanfi, Clémentine Hugol Gential, Stephanie

Chambaron, Corinne Buisson, Virginie van Wymelbeke, Claire Sulmont-Rossé

\section{To cite this version:}

Marie-Claude Brindisi, Ousrat Chanfi, Clémentine Hugol Gential, Stephanie Chambaron, Corinne Buisson, et al.. Quelle information accompagne la distribution des Compléments Nutritionnels Oraux (CNO) à l'hôpital: un état des lieux. Journées Francophones de Nutrition (JFN), Nov 2019, Rennes, France. 1 p., 2019. hal-02788059

\section{HAL Id: hal-02788059 \\ https://hal.inrae.fr/hal-02788059}

Submitted on 5 Jun 2020

HAL is a multi-disciplinary open access archive for the deposit and dissemination of scientific research documents, whether they are published or not. The documents may come from teaching and research institutions in France or abroad, or from public or private research centers.
L'archive ouverte pluridisciplinaire HAL, est destinée au dépôt et à la diffusion de documents scientifiques de niveau recherche, publiés ou non, émanant des établissements d'enseignement et de recherche français ou étrangers, des laboratoires publics ou privés. 


\title{
QUELLE INFORMATION ACCOMPAGNE LA DISTRIBUTION DES COMPLÉMENTS NUTRITIONELS ORAUX (CNO) À L'HÔPITAL ? UN ÉTAT DES LIEUX
}

\author{
M-C Brindisi, ${ }^{1,2}$, O Chanfi ${ }^{1}$, C Hugol-Gential ${ }^{3}$, S Chambaron ${ }^{1}$, C Buisson $^{2}$, \\ V Van Wymelbeke ${ }^{1,2}$, C Sulmont-Rossé $^{1}$ \\ ${ }^{1}$ CSGA, AgroSup, CNRS, INRA, Univ. Bourgogne Franche-Comté, F-21000 Dijon, France \\ ${ }^{2}$ CHU François Mitterrand, Centre Gériatrique Champmaillot, F-21000 Dijon, France \\ ${ }_{3}^{3}$ Université de Bourgogne, Laboratoire CIMEOS (EA4177), Maison des Sciences de I'Homme de Dijon.
}

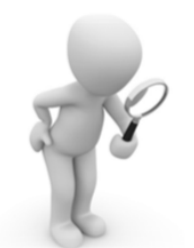

\begin{abstract}
OBSERVATION
Un observateur neutre a suivi les équipes de service et consigné tous les échanges verbaux entre soignants et patients lors de la distribution des CNO.
\end{abstract}

Au total, 221 observations ont été réalisées dans 5 services hospitaliers ( $n=90$ patients, dont $69 \%$ de plus de 65 ans).

\begin{tabular}{|c|c|c|}
\hline & $\%$ & Exemples \\
\hline Rien & $41 \%$ & Le personnel dépose le plateau sans rien dire à la personne \\
\hline Service hôtelier & $43 \%$ & " Bon appétit ! ", " Voilà pour vous " \\
\hline Produit & $6 \%$ & " Je vous apporte votre boisson de l'après-midi " \\
\hline Marque & $4 \%$ & " Voici votre petit Fortimel " " Votre Fortimel, monsieur " \\
\hline Fonction du CNO & $3 \%$ & $\begin{array}{l}\text { "C'est la boisson enrichie, il faut la boire " } \\
\text { "Ce sont des boissons hyperprotéinées " }\end{array}$ \\
\hline Autres & $3 \%$ & $\begin{array}{l}\text { "Le Délical, vous ne l'avez pas bu ce matin ?" } \\
\text { "Essayez de les prendre !" }\end{array}$ \\
\hline
\end{tabular}

Dans $84 \%$ des cas, le CNO est apporté au patient sans aucune référence verbale au produit. Le CNO est posé sur le plateau du petit-déjeuner ou déposé dans la chambre lors des collations sans aucun mot permettant au patient de repérer le CNO comme un élément distinctif des aliments qui composent le plateau. Dans $74 \%$ des observations réalisées, le CNO est ramassé sans interroger le patient sur sa consommation

\section{ENQUÊTE}

Un expérimentateur a interrogé 41 patients dans le cadre d'un entretien semi-directif (61\% de femmes ; 65-94 ans ; âge moyen : 79 ans).

Des questions ouvertes et fermées portaient sur la représentation des CNO : c'est quoi ? À quoi ça sert ?

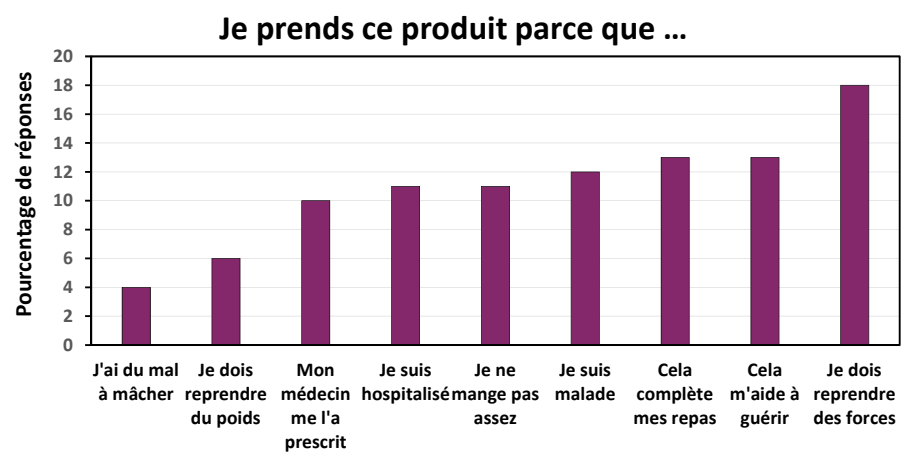

Au cours de la phase d'enquête, $66 \%$ des patients déclarent ne pas avoir reçu d'information sur le CNO. Pour $71 \%$ des patients, le CNO est un " complément alimentaire " qui permet de " compléter/rééquilibrer l'alimentation " (39\%) ou " d'aller mieux » (34\%). Seuls $29 \%$ des participants l'associent à une perte d'appétit, de poids ou à la dénutrition et $27 \%$ ne savent pas du tout à quoi sert ce produit.

Notre étude souligne le manque d'information donnée aux patients concernant les objectifs thérapeutiques et les bénéfices cliniques attendus des CNO, ce qui peut au moins partiellement expliquer la faible observance associée à ces produits. Le moment de la distribution devrait être un moment clé permettant la diffusion de ces informations.

$\begin{gathered}\text { Tenez, la petite boisson } \\ \text { de l'après-midi! }\end{gathered}$
Madame!

